\title{
INVESTIGACIONES
}

\section{Poder, ideología y conocimiento: aperturas epistemológicas del concepto de universidad en Chile*}

\author{
Power, ideology and knowledge: Epistemological apertures \\ of the concept of university in Chile
}

\section{Nicolás Díaz Barrera ${ }^{a}$, Iván Oliva Figueroa ${ }^{b}$}

${ }^{a}$ Centro de Investigación en Educación. Universidad Bernardo O’Higgins. Correo electrónico: ndiaz.lenguaje@gmail.com

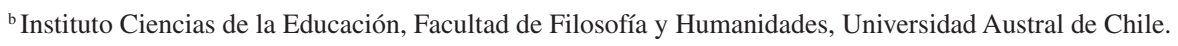
Correo electrónico: ivanoliva@uach.cl.

\begin{abstract}
RESUMEN
El siguiente reporte de investigación indagó, desde un enfoque cualitativo, en la identificación de diversas formas de institucionalización del conocimiento en la universidad través de la metodología de Análisis Crítico del Discurso. Esta metodología fue aplicada a diversas prospectivas de Misión-Visión de veinticuatro (24) universidades chilenas, divididas en estatales, privadas y particulares con aporte estatal. Utilizando un constructo teórico basado en complejidad, transdisciplina, ideología y poder, se codificó desde la base de tres (3) categorías generales (Poder, ideología y conocimiento) mediante el soporte informático Atlas.ti versión 5.0. Posteriormente, fue elaborado un modelo de red que permite observar a nivel general y específico la densidad de asociaciones entre códigos, atendiendo a sus diversos contextos de producción. Los principales resultados indican que una fuerte presencia de la ideología se manifiesta es estas prospectivas a través de elementos discursivos que evidencian el ejercicio del poder mediante mecanismos institucionales y sociales.
\end{abstract}

Palabras clave: misión visión institucional, complejidad, transdisciplina, educación terciaria.

\begin{abstract}
The following research report investigated, from a qualitative approach, the identification of several forms of legitimation of power, ideology, and institutionalization of knowledge in Chilean higher education through the methodology of Critical Discourse Analysis developed by Teun van Dijk. This methodological resource was applied to the diverse Mission-Vision prospects of 24 universities in Chile, divided into state, private, and private with state contribution. Using a theoretical construct based on complexity, transdiscipline, ideology, and power, encoded results were obtained in 3 general categories (power, ideology and knowledge) through the computer support Atlas.ti version 5.0. Subsequently, a network model was developed, allowing the observation, at both general and specific levels, of the density of associations between codes, attending to their diverse contexts of production. The main results indicate that a strong presence of ideology is manifested through these prospects through discursive elements that demonstrate the exercise of power through institutional and social mechanisms.

Keywords: institutional mission vision, complexity, transdiscipline, tertiary education.
\end{abstract}

\footnotetext{
El articulo forma parte del programa de investigación asociado al proyecto Fondecyt-Regular 1150923 "Interfaces universidad sociedad desde la prospectiva transdisciplinaria: metodología de redes complejas y minería de datos aplicadas a dinámicas de organización del conocimiento a escala social".
} 


\section{INTRODUCCIÓN}

Se ha propuesto este trabajo desde un punto de vista reflexivo y crítico con sustrato en los conceptos de poder, ideología y conocimiento esbozados por los autores Michel Foucault, Teun van Dijk y Edgar Morin respectivamente. Esta elección se sostiene sobre la base de la necesidad de cuestionar los fundamentos epistemológicos de la Educación Superior chilena (en adelante ES) desde campos disciplinarios diversos y simultáneamente convergentes a las ciencias de la educación.

En este sentido, Morin es quien proporciona una mirada más directa al fenómeno educativo, pero desde una propuesta epistémica y transdisciplinaria que involucra decididamente la inclusión de diversas disciplinas en la resolución de conflictos globales y específicos (Morin 2001, 2006a). Morin reformula el concepto de conocimiento y lo lleva a un orden cualitativo y organizacional, develando la importancia de que las diversas disciplinas generen una mirada crítica de sus propias parcelas de saber como manera de cuestionar sus propios fundamentos (Espina 2007) epistemológicos (Espina 2007) y a la vez, la importancia de vincular metodologías y epistemologías que conlleven una mayor complejidad en los procesos de construcción de conocimiento.

No es nuestro interés hacer tabula rasa con los elementos anteriores a esta investigación, sino buscar espacios de confluencia que abran puertas a nuevas indagaciones más allá del financiamiento de la educación, las estructuras de organización, la búsqueda de la innovación y la calidad que han sido temas ya tratados con frecuencia durante las dos últimas décadas. Nuestro principal objetivo es plantear la posibilidad de un campo de estudio transdisciplinar desde donde emerjan nuevas problemáticas antropo-sociales, con mayores grados de complejidad cualitativa, a la vez de configurar elementos de resolución y prospectiva basados en la socialización y organización multinivel de metodologías e isomorfismos teóricos.

Desde esta base, Teun van Dijk plantea en su concepto de ideología esta necesidad de involucramiento de las disciplinas; una aproximación multidisciplinaria del fenómeno en cuestión. van Dijk plantea la posibilidad de un concepto de ideología desde la lingüística asociada a la psicología y la neuro-cognición, primer atisbo transdisciplinario desde la propuesta teórica de Ricoeur en Ideología y utopía (Ricoeur 1994).

Foucault a su vez, contribuye a nuestra aproximación por su concepción del poder como un fenómeno no estático, que existe solamente en su ejercicio, al contrario de los simbolismos utilizados por otros autores que asocian el poder a la postura jerárquica de quien lo posee y lo hereda, evidenciando que una microfísica del poder se presenta en espacios locales, incluso hasta en el cuestionamiento del individuo en sí (Foucault 1966). Desde esta base, se hace patente la necesidad de visualizar al individuo como un cuerpo y una cognición sometidas a discursos que lo transforman en su devenir y que confluyen, en este aspecto, a las propuestas de van Dijk sobre la triada discurso-sociedad-cognición.

Somos conscientes de las diversas escuelas de las cuales provienen estos autores, quienes en algunos aspectos hasta podrían arriesgar grados de inconmensurabilidad y resquebrajar las bases teóricas de esta investigación, no obstante también se asume el reto de generar una prospectiva transdisciplinaria que considere recursos epistémicos y metodológicos derivados de otras disciplinas, que a un cierto nivel de profundidad, conllevan isomorfismos teóricos pertinentes a las ciencias de la educación y sus procesos de interpretación. 


\section{UNIVERSIDAD: DESDE LO GLOBAL A LO LOCAL}

La Universidad saca a la luz todas las capacidades, incluida la incapacidad.

Antón Pavlovich Chéjov

Lemaitre caracteriza a la institución universitaria globalizada como un organismo colonizador de la calidad. Se constituye y legitima a través de un discurso político situado en las nociones de desarrollo, innovación y excelencia, lo que provoca el enclaustramiento intelectual del conocimiento que proviene y se gesta en sociedad (Lemaitre 2005).

En este contexto y en el ámbito específico de la educación superior, las premisas de calidad quedan a disposición de elementos externos tanto de la sociedad como del mundo académico que se someten a la regulación económica de su acción. Gandarilla menciona que la educación superior hoy es una experiencia de masas, los problemas disciplinarios ceden el paso a la investigación orientada por el mercado, y las ideas generales acerca del mundo y la historia pierden cada día más peso. Lo anterior conlleva una dureza implícita en el diagnóstico del estado de la educación superior chilena, que ha sido relegada, reconfigurada y capturada por las diversas dimensiones económicas y socio-políticas del modelo capitalista (Gandarilla 2010). Unesco menciona que "las economías más avanzadas hoy día se basan en la mayor disponibilidad de conocimiento. Las ventajas comparativas dependen cada vez más del uso competitivo del conocimiento" (Unesco 2011). Esta centralidad del fenómeno del conocer lo convierte en un pilar fundamental de la riqueza y el poder de las naciones, pero, a la vez, estimula la tendencia a su consideración como simple mercancía, sujeta a las reglas del mercado y susceptible de apropiación privada. Es más, el concepto de capitalismo académico (Fonti 2014) refiere a todo tipo de acción para atraer recursos a la institución universitaria (actividad mercantil) y conductas de este organismo basado en el sistema de mercado. Lo anterior es el panorama general que se observa en el Estado del Arte de estudios sobre educación (Brunner 2000).

Conforme a Jessop, la globalización, desde un punto de vista estructural, se refiere a la creación y desarrollo de interdependencia global entre las acciones, organizaciones e instituciones de la economía, la política, el derecho, amén de otros (sub)sistemas funcionales y el mundo de la vida. En cambio, desde consideraciones estratégicas y de agencia, la globalización guarda relación con los diferentes intentos de los actores de coordinar globalmente sus actividades entre los diferentes subsistemas funcionales y el mundo de la vida (Jessop 2008).

Brunner menciona que, desde el origen de la universidad, esta se ha situado en un campo de fuerzas entrecruzadas que se expresa mediante el lenguaje del poder y el derecho. Sitúa este lenguaje como un poder contestado, es decir adquirido mediante negociaciones, a través de la razón y la astucia cuyo fin en esencia es constituirse como un elemento institucional a la vez que intelectual. Esta institucionalidad daba a quienes pertenecían a ella de un prestigio de libertades, privilegios e inmunidades, otorgadas por cargos académicos, la enseñanza y el conocimiento (Brunner 2000).

De esta manera, Brunner caracteriza dos modelos resultantes de los nuevos intereses y pretensiones que la sociedad manifestaba como proyección de la universidad, a saber, el napoleónico y el humboldtiano. El primero impuso a las antiguas corporaciones la 
disciplina de las burocracias, profesionalizó la carrera funcionaria de los académicos y convirtió a las universidades en objetos de las políticas nacionales de educación. El segundo les proporcionó un nuevo significado a su labor educativa, proponiendo el cultivo del nuevo conocimiento en los límites de las disciplinas, en un ambiente caracterizado por las libertades de enseñar y aprender (Brunner 2000).

A esto se suma la identificación que hace Fonti respecto a que la división del trabajo académico se vuelve más compleja y densa, mayor la especialización disciplinaria, más gravitante el peso de la investigación y las ciencias (Fonti 2014). Estos rasgos son los que subyacen hoy en día en la subdivisión de facultades, institutos, carreras y mallas curriculares. La fragmentación disciplinaria emerge a causa de la densa gama de diversidades a la par de la especificidad que cada una de estas requería en su estudio sistemático (Gandarilla 2010).

A esto agregamos también las relaciones de poder generadas en todo tipo de organización social con estamentos jerárquicos y la aparición de un nuevo fenómeno, Lemaitre menciona que existe una imposición de cultura, de prioridades políticas y económicas, pero lo que no hay es un enemigo identificable o deseo explícito de conquista (Lemaitre 2005). Llamamos a este fenómeno globalización y nuestra reacción tiene elementos ambivalentes frente a ella. Esta ambivalencia respecto a la globalización se deposita en la manera en que las prioridades políticas y económicas son tratadas por los estamentos gubernamentales, alejadas del contexto de las problemáticas y con lineamientos poco específicos respecto a la dimensión cualitativa de desarrollo que persiguen. En definitiva, pese a utilizar esta noción o categoría maestra en gran parte de sus lineamientos, pocas veces es examinada epistemológicamente, atendiendo a las estructuras de significación latentes a su definición teórica y operativa.

\section{IDEOLOGÍA: HACIA UNA VISIÓN COMPLEJA Y TRANSDISCIPLINAR DEL FENÓMENO}

Utilizaremos el siguiente corolario de Foucault como adagio para situar el fenómeno de la ideología en torno al discurso:

los códigos fundamentales de una cultura -los que rigen su lenguaje, sus esquemas perceptivos, sus cambios, sus técnicas, sus valores, la jerarquía de sus prácticas- fijan de antemano para cada hombre los órdenes empíricos con los cuales tendrá algo que ver y dentro de los que se reconocerá (Foucault 1966).

Estos códigos serán entendidos desde la lógica de las "prácticas sociales discursivas" (van Dijk 1999) como elementos de producción y reproducción de signos y símbolos. En este contexto, la manera de entender la relación entre cognición, discurso y sociedad se sitúa desde la ideología como principal fuente productora de relaciones simbólicas, un sistema de ideas que se estructura a partir de la cognición y sus interacciones socio-comunicativas mediante la práctica social del discurso proyectada en la sociedad, entendiendo que "aunque los discursos no son las únicas prácticas sociales basadas en la ideología, son efectivamente las fundamentales en su formulación y, por tanto, en su reproducción social" (van Dijk 1999), cotejando la utilización del lenguaje, el texto, la conversación y la comunicación (que se incluyen en el término de discurso) y la modificación desde las 
dimensiones mentales, las situaciones y estructuraciones sustanciales del organismo social.

Sobre la ideología, van Dijk proporciona la siguiente perspectiva: es la base de las representaciones sociales compartidas por los miembros de un grupo y que les permiten organizar la multitud de creencias sociales acerca de lo que sucede, bueno o malo, correcto o incorrecto, según ellos, y actuar en consecuencia (van Dijk 1999). Ampliado por Stuart Hall en van Dijk, estas adquieren el estatus de estructuras mentales -lenguajes, conceptos, categorías, imágenes del pensamiento y los sistemas de representación- que diferentes clases y grupos sociales despliegan para encontrarle sentido a las formas en que la sociedad funciona, para explicarla y hacerla inteligible (Hall 1984; van Dijk 1999).

Morin, en consonancia con van Dijk, también hace patente que la ideología al ser un sistema de ideas se constituye por una constelación de conceptos asociados de forma solidaria y cuya disposición es establecida por los vínculos lógicos en virtud de axiomas, postulados y principios de organización subyacentes; tal sistema entonces, producirá en su campo de competencia enunciados con un valor de verdad y, eventualmente, predicciones sobre todos los hechos y eventos que en él deben manifestarse. Desde la base de lo anterior, se genera una lógica sistémica que tiende a la clausura de sus fronteras en la cual las ideas poseen su consistencia únicamente dentro de un sistema que sea capaz de integrarlas (Morin 2001, 2006a; van Dijk 1999, 2008, 2012).

Los elementos planteados por Morin, van Dijk y Foucault nos sirven de entrada para la proposición de ideología esbozada y que utilizamos como referencia en esta investigación (Morin 2001; van Dijk 1999, 2008; Foucault 1966). La ideología cumple una función primordial que consiste en asegurar la articulación simbólica de la sociedad en sus variadas formas (política, religiosa, etc.) la cual puede convertirse en la expresión deformadora de los intereses de una clase como lo pretende la tradición marxista; o bien, puede actuar también como una instancia de legitimación. Esta permite cubrir el vacío que se genera entre la pretensión de las autoridades políticas de que su autoridad sea considerada legítima por parte de la ciudadanía y la percepción que esta última tiene de la mayor o menor legitimidad que le asiste a la autoridad para exigir semejante reconocimiento. De esta manera la ideología asociada al constructo de las universidades modernas, permite asegurar en algún grado la legitimidad del ejercicio del poder sobre la ciudadanía, quien también reconoce este como legítimo.

\section{PODER Y UNIVERSIDAD}

Las líneas de investigación de Michel Foucault según Márquez se centran en el estudio de la locura, del humanismo y de las prisiones procediendo con el método genealógico, que implica el análisis que da cuenta de su emergencia y de su procedencia, más que de su historia. Simultáneamente, su trabajo consiste en crear una historia de los diferentes modos por los cuales, en nuestra cultura, los seres humanos se convierten en sujetos Mediante esto genera tres condensaciones globales para su pensamiento: el problema del saber, el problema del poder y el problema de la subjetivación (Márquez 2007).

Una de las interrogantes principales que Foucault intenta responder es la posibilidad de que el poder pueda deducirse de la economía. De esta manera elabora dos concepciones de poder en la historia: una, la concepción jurídica y liberal del poder político, encontrada en los filósofos del siglo XVIII; y otra, la concepción marxista a la que da el nombre de 
funcionalidad económica del poder. El poder, considerado como funcionalidad económica, se puede interpretar en que consiste en esencia en mantener relaciones de producción de modo que las fuerzas productivas constituyan una dominación de clases.

Foucault nos expresa algunos ribetes sobre su noción de poder: "por poder no quiero decir 'el poder', como conjunto de instituciones y aparatos que garantizan la sujeción de los ciudadanos en un Estado determinado. Tampoco indico un modo de sujeción" (Foucault 1998). Otra definición encontrada es esta:

Por poder, me parece que es necesario comprender, por de pronto, la multiplicidad de las relaciones de fuerza que son inmanentes al dominio donde ellas se ejercen, y que son constitutivas de su organización; el juego que por la vía de las luchas y enfrentamientos incesantes las transforma, las refuerza, las invierte; los apoyos que estas relaciones de fuerza encuentran unas en otras, y que las lleva a formar cadenas y sistemas, o, al contrario, los desencuentros, las contradicciones que las aíslan entre sí; las estrategias, en fin, en las cuales cobran efecto, y cuyo diseño institucional toma cuerpo en los aparatos estáticos, en la formulación de la ley, en las hegemonías sociales. (Foucault 1998).

En este orden de ideas, siguiendo a Márquez, la perspectiva del poder es ejercida positivamente sobre la vida, procurando administrarla y multiplicarla. Sobre ella ejerce controles precisos y manipulaciones por medio de la regulación, construyendo una anatomía política o una biopolítica, que se ejerce sobre el individuo, pero fundamentalmente sobre las poblaciones (Márquez 2007). Mediante las sociedades disciplinarias (definidas como aquellas sociedades que ponen en marcha dispositivos de control que producen y regulan costumbres hábitos y prácticas como se verá más adelante) se colocan en función las tácticas de poder que dan su máximo de identidad produciendo un crecimiento y un rendimiento efectivo de los aparatos en el interior de los cuales se ejerce: aparatos pedagógicos, aparatos militares, aparatos industriales, aparatos médicos (Márquez 2007). En principio, esto obedecía a circunstancias históricas como el crecimiento demográfico del siglo XVIII, que exigía fijar la población flotante; y, segundo, al crecimiento del aparato productivo que exigía hacer crecer su rentabilidad, es decir, de lo que se trataba era de intentar correlacionar población y producción (Márquez 2007). Estos devienen en lógicas de interacción normal entre la institución y el individuo, y su cuestionamiento se produce en formas aleatorias y en contextos específicos.

Según Foucault, el poder se constituye desde abajo, desde microprácticas. Esta mirada hacia lo micro demostró cómo muchas experiencias que quedaron fuera del análisis del poder (la locura, la sexualidad, el encierro, la criminalidad) se sitúan en el seno del campo de lo político De esta noción se desprende el primer atisbo de la "microfísica del poder" (Foucault 2001).

Como síntesis de este segmento, nos orientamos sobre los tres ejes de acción ejercida desde algunos sectores del poder en el cuerpo y, por tanto, en la cognición, a saber: a) una intervención sobre el cuerpo con el fin de que este produzca signos que se manifiesten en signos de respeto, devoción, sujeción o servidumbre; b) un uso del cuerpo como espacio simbólico de expresión de un poder político soberano que tiene derecho sobre la vida y la muerte; y c) una utilización del cuerpo en el plano del trabajo. Un bio-poder en cuya configuración y legitimación convergen las más diversas disciplinas (medicina, psicología, 
biología, pedagogía, psiquiatría) y que se manifiesta a la vez como una política del cuerpo encargada de educarlo, aumentar sus aptitudes, maximizar su fuerza y su utilidad, y también como una planificación global de la población o bio-política encargada de regular la proliferación de nacimientos, la mortalidad y los niveles de salud y longevidad.

Desde esta base y desde una óptica compleja, sostenemos que el constructo semiótico asociado a la universidad moderna conlleva una huella matricial y hologramática que condensa muchos de los aspectos hasta aquí tratados (Giraldo 2005). Entendemos que la institución universidad no es un subsistema social, sino más bien una instancia dialógica y recursiva que es producto y productor de la sociedad en la cual se inscribe, opera y se legitima.

En este sentido, los objetivos de la presente investigación pueden sintetizarse en interpretar y comprender las formas de legitimación de poder, manifestaciones de ideología e institucionalización del conocimiento a través del Análisis Crítico de Discurso aplicado a fuentes primarias emanadas de universidades estatales, privadas y particulares con aporte estatal.

De manera específica, los objetivos buscan: a) establecer un constructo teórico conceptual desde el derrotero epistemológico de Morin, van Dijk y Foucault para posibilitar instancias críticas de apertura/clausura epistemológica de la Educación Superior en Chile; b) interpretar elementos de legitimación del poder, ideología e institucionalización del conocimiento a través de la proposición analítica de van Dijk, aplicada a diversas fuentes primarias y c) Generar una prospectiva compleja de la ESC en base a los resultados obtenidos del análisis de las fuentes primarias con el propósito de discutir la presencia de las nociones de poder, universidad, ideología y conocimiento en el discurso académico institucional.

\section{METODOLOGÍA}

La naturaleza y el sustrato desde el cual emerge la interpretación de los fenómenos se encuentra bajo el paradigma cualitativo de investigación y se sostiene mediante análisis y explicaciones que abarcan la comprensión de la complejidad, el detalle y el contexto (Vasilachis 2006). El tipo de diseño corresponde a exploratorio (con elementos descriptivos) cuyo objetivo es aumentar el grado de familiaridad con fenómenos relativamente desconocidos, obtener información sobre la posibilidad de llevar a cabo una investigación más completa sobre un contexto particular de la vida real, investigar problemas del comportamiento humano que se consideren cruciales para profesionales de determinada área, identificar conceptos o variables promisorias, establecer prioridades para investigaciones posteriores o sugerir afirmaciones (postulados) verificables (Flick 2004).

La elección del corpus para esta investigación, según criterios de Miles y Huberman, se basa en un tipo de muestreo cualitativo no probabilístico de máxima variación, cuyo propósito es documentar diversas variaciones identificando patrones comunes importantes. Estos patrones comunes serán los conceptos de ideología, poder y conocimiento (Miles y Huberman 1994).

Se utilizaron veinticuatro (24) fuentes primarias documentales que involucran la Misión y Visión de instituciones universitarias chilenas. La Misión es el motivo, propósito, fin o razón de ser de la existencia de una organización porque define 1) lo que pretende 
cumplir en su entorno o sistema social en el que actúa, 2) lo que pretende hacer, y 3) el para quién lo va a hacer; y es influenciada en momentos concretos por algunos elementos como la historia de la institución, los factores externos o del entorno, los recursos disponibles, y sus capacidades distintivas. La Visión, por su parte, se define como una exposición clara que indica hacia dónde se dirige la empresa a largo plazo y en qué se deberá convertir, tomando en cuenta el impacto de las nuevas tecnologías, de las necesidades y expectativas cambiantes globales y específicas.

Dichos documentos fueron divididos en universidades estatales (8) universidades privadas (8) y universidades particulares con aporte estatal (8) todos de una extensión mínima de media plana y máxima una plana. La relevancia de la utilización de estos documentos para la presente investigación radica en que los diversos contextos donde se producen los discursos difieren en el énfasis a los conceptos utilizados en esta investigación, por lo que la discusión se enriquece en la medida que los argumentos y posicionamientos se amplían cualitativamente para observar el fenómeno en cuestión en relación al marco teórico presentado.

\section{RESULTADOS}

\subsection{TIPOLOGÍAS Y PRIMER NIVEL DE ASOCIACIÓN}

\subsubsection{Ideología}

El fuerte lazo manifestado entre las instituciones y las empresas, mayoritariamente en las universidades privadas y particulares con aporte estatal se relaciona con la aparición de un nuevo modelo económico, una mayor demanda de conocimiento manifestada por la población y la globalización, que genera la inclusión del saber a estructuras de producción económicas, donde la educación se reacomoda en la matriz social como un bien intercambiable por dinero, accesible en la medida que se posean los recursos, pero ya no limitada (teóricamente) a las elites intelectuales, si no abierta a un rango más amplio a diferencia de los años anteriores cuando se ofrecía un privilegio más que un servicio. De la misma manera, el conocimiento entra en un mercado de producción válido en la medida que obedezca a prioridades sociales, que genera la disyuntiva en las universidades de incorporarse a estas nuevas redes de saber o aislarse junto a los paradigmas tradicionales universitarios.

Respecto a la religión fuertemente representada en este código (Regnum Cristi, Iglesia católica, Humanismo, Opus Dei) van Dijk menciona que los sistemas de creencias son productos del pensar, por lo que el conocimiento es solo una categoría específica de estos sistemas en los que "nosotros (como grupo, comunidad, cultura, caso particular o institución) consideramos 'creencias verdaderas' de acuerdo con ciertos fundamentos o criterios (de verdad)" (van Dijk 1999). Estos criterios son válidos, correctos, certificados y sostenidos de una manera general cuando reúnen ciertos estándares de verdad socialmente compartidos las cuales, en este caso, son las religiones. En un nivel mayor encontramos a las instituciones que manejan o propagan esas cogniciones, acciones, interacciones y relaciones de grupo. Van Dijk entenderá las instituciones como contrapartida práctica o social de las ideologías, del mismo modo en que las ideologías organizan la cognición de 
grupo, las instituciones organizan las prácticas y a los actores sociales (van Dijk 1999). Por tanto la religión, como un sistema de creencias, se encuentra en igualdad de condiciones para propagar un discurso de formación profesional, en la medida que posee la capacidad de generalizar los acontecimientos que producen los procesos de inferencia personales. Los acontecimientos que generan casos y las representaciones generales socialmente compartidas producen modelos mentales que interactúan de forma dialógica con el discurso (interpretaciones, asociaciones, analogías) que están en constante interacción con el vasto conocimiento previo que es activado a través de la memoria episódica.

\subsubsection{Poder}

En los documentos analizados se hace patente la formación de profesionales para ingresar inmediatamente al campo laboral lo cual se asocia a una de las interrogantes principales que Foucault intenta responder que es la posibilidad de que el poder pueda deducirse de la economía.

En consecuencia, nos adherimos al enunciado sobre el poder para Foucault que se instala bajo lógicas llamadas de normalización donde el espectro de análisis y reproducción se globalizan desde esta óptica: "la imposición de competencias en escuelas y universidades, el enciclopedismo y memorización, en la interpretación de una realidad bajo una coacción que soslaya la percepción de los estudiantes limitando su creatividad" (Ávila-Fuenmayor 2007). Foucault es claro en aseverar que "en todo lugar donde hay poder, el poder se ejerce. Nadie es su dueño o poseedor, sin embargo, sabemos que se ejerce en determinada dirección; no sabemos quién lo tiene, pero sí sabemos quién no lo tiene" (Foucault 2001). El poder es una magnitud no estática, en la cual las relaciones de fuerza están en continuo cambio: son estados de poder, locales e inestables, que lo hacen omnipresente.

El poder está por todas partes; no es que lo englobe todo, es que viene por todas partes. Y el poder, en aquello que tiene de permanente y de repetitivo, de inerte, de autorreproductor, no es sino el efecto del conjunto, que se dibuja a partir de todas estas movilidades. (Foucault 1976).

El poder es sinónimo de la creación de cuerpos dóciles, preparados para responder a estímulos más que a generarlos, a prestar servicios e insertarse en la máquina social de forma inmediata.

\subsubsection{Conocimiento}

El desglose de lo anterior nos indica que las disciplinas pueden ser consideradas dominios cognitivos que acotan su accionar a sus dinámicas de identidad. Estos dominios tienden a plegarse sobre sí mismos, lo que genera una frontera epistemológica en base a lenguajes y teorías de relativa autorreferencia y establece los parámetros tautológicos en los que cae la disciplina en sí (tanto como concepto en su estructura y a la vez como elemento de configuración semiótica en su fondo).

En el análisis nos encontramos con tres concepciones resumidas de conocimiento, a saber a) un conocimiento complejo, cuyas líneas se dirigen hacia la integralidad de distintos 
conocimientos, a diálogos inter y transdisplinarios, y una fuerte relación con el entorno; b) un conocimiento mercantil, una tecnocratización de las competencias adquiridas para ser aplicadas de forma inmediata en el mercado laboral y generar productividad; y c) un conocimiento avanzado, que se visualiza en la continuidad de estudios de postgrado y de investigación.

Así entonces, a la luz de los análisis, es necesario hablar de conocimientos y de realidades que se presentan desde la multiplicidad de enfoques complejizados, según la perspectiva de Díaz, Soler y Oliva, donde la diversidad de aprendizajes y saberes nos llevan a una diversidad de perspectivas desde las cuales percibimos el mundo, más allá de una concepción acumulativa de saberes que han puesto al conocimiento dentro de variables cuantificables (Díaz, Soler y Oliva 2013). Nos adherimos a Toledo quien comprende en un primer nivel que los sujetos que comparten un mismo acervo social de conocimientos y experiencias son provistos, por medio de su familiarización con los hábitos compartidos, de una competencia interpretativa a partir de la cual, escapando del núcleo autorreferencial del ser humano hacia su propio actuar hace posible comprender las pautas de interacción de los otros, sus motivos y causas, en virtud de lo cual, los meros individuos se convierten en auténticos actores sociales (Toledo 2007). Estas categorías las encontramos presentes tanto en universidades estatales y, en menor medida, en universidades privadas.

Como conclusión, las descripciones que aquí se hacen respecto a la visión compleja de la realidad propuesta por Edgar Morin, puede constituirse como un enfoque pertinente dentro de las innovaciones educativas de este último tiempo (Morin 2001, 2006b). Una visión compleja de la universidad como la descrita anteriormente, favorece la reflexión en torno a la distintas disciplinas existentes en ella, lo que posibilita el intercambio y el diálogo necesario hoy en día, sin anteponer el peligro a perder la identidad disciplinar generada desde un enfoque local clausurado, tomando los elementos que esto puede generar a nivel macro en la construcción de un conocimiento colectivo y participativo, y a nivel micro, la comprensión del mundo como un todo sin necesariamente reducirlo a las características de sus partes y favoreciendo las facultades asociativas de los estudiantes con los elementos presentes en los enfoques de la realidad. De esto se desprenden un segundo, tercer y cuarto nivel de asociaciones simétricas, asimétricas y transitivas que profundizan en los planos de tendencia discursiva mediante redes cognitivas emanadas del análisis mediante Atlas.ti.

\section{DISCUSIÓN DE RESULTADOS POR NIVELES DE ASOCIACIÓN}

\subsection{SEGUNDO NIVEL DE ASOCIACIÓN}

Entendiendo que un primer nivel de asociación corresponde a los conceptos de Conocimiento, Poder e Ideología, un segundo nivel de análisis asociativo nos permite entre otras cosas, visualizar el entrecruce de las citas con los códigos pero dificulta las relaciones simétricas o asimétricas de causalidad entre los códigos. Si en un tercer nivel de asociación somos capaces de observar la asociación entre códigos y citas, en un segundo nivel se hacen visibles las confluencias de los códigos con las citas, pero no una asociación directa entre los códigos, tal como sucede en el siguiente ejemplo (ver Figura 1): 
Figura 1. Segundo nivel de asociación mediante análisis de unidad hermenéutica

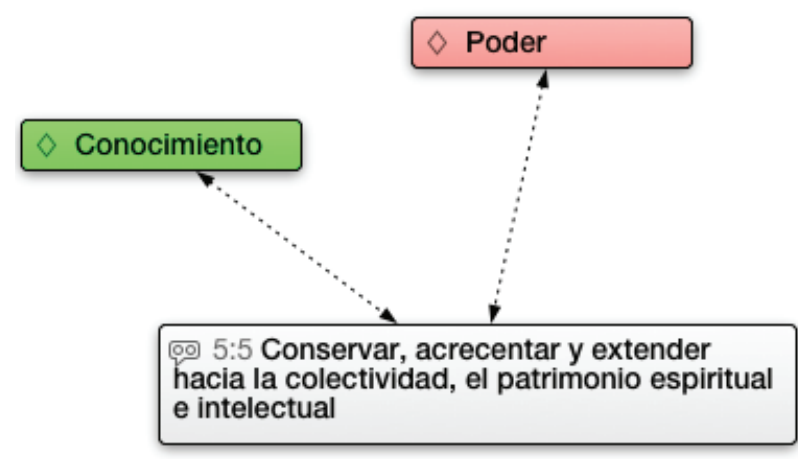

Fuente: elaboración propia

Se encuentra patente la existencia de elementos propios del conocimiento y el poder en la cita utilizada, pero se dificulta realizar una asociación entre los códigos ya que la cita no da luces sobre cómo podrían interferir estos dos conceptos, solo se los categoriza y se los presenta como una densidad involucrada en la unidad hermenéutica, pero realizar una asociación entre los códigos sería forzar sus asociaciones.

Se asocia el código de universidad al de ideología en la medida que la institución universitaria se autodefine como católica (sistema de creencia según van Dijk 2008) pero no se hace patente la posibilidad de redefinir o asociar los dos códigos ya que la cita no es lo suficientemente explícita para poder indicarnos si una es causa de la otra o viceversa. Otro ejemplo similar de esto el siguiente (ver Figura 2):

Figura 2. Ejemplo de segundo nivel de asociación mediante análisis de unidad hermenéutica

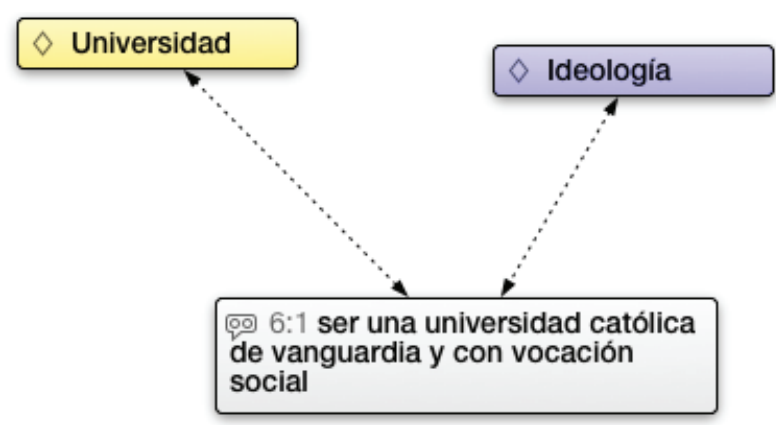

Fuente: elaboración propia 


\subsection{TERCER NIVEL DE ASOCIACIÓN}

En este nivel nos encontramos con la asociación entre códigos y citas, más explícitamente, con la asociación de códigos y sus causalidades o asociaciones, como ya pudimos observar al comienzo de este apartado. Durante la indagación nos encontramos con este ejemplo (ver Figura 3):

Figura 3. Tercer nivel de asociación mediante análisis de unidad hermenéutica

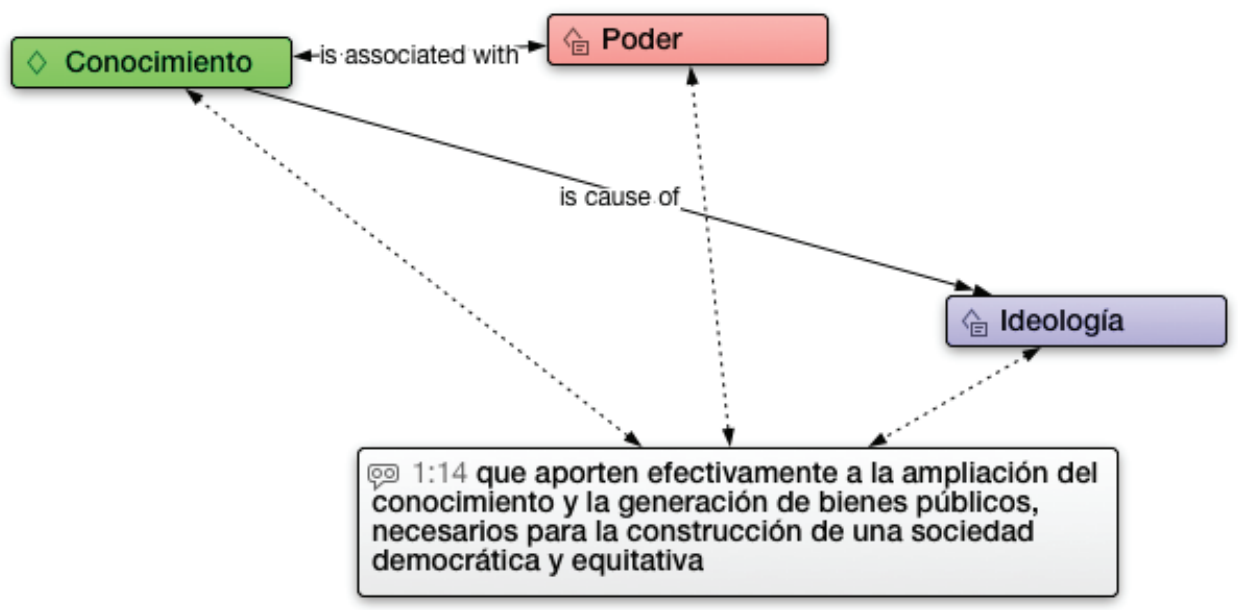

Fuente: elaboración propia

La asociación entre conocimiento es simétrica y confluyen en la cita representada anteriormente. Se instala un tercer nivel que se desprende del código conocimiento el cual es la ideología como causa directa y explicativa de los elementos implícitos en la cita, mientras que se asocia el poder con el conocimiento, elevando el nivel de complejidad manifestado en los análisis previos. ¿Qué significa que estos códigos estén asociados? Significa que según los enunciados de Foucault y los autores de este trabajo, el conocimiento está presente mientras se habla de poder (la generación de bienes públicos) y que el poder está relacionado al conocimiento en la medida que brinda los elementos de los cuales el conocimiento debe hacerse cargo, como la elaboración de un campo del saber, la orientación (formal o informal) de proyectos de investigación, etc.

\subsection{CUARTO NIVEL DE ASOCIACIÓN}

En este nivel nos encontramos con una densidad de asociación que supera (o profundiza) las categorías anteriormente analizadas complementadas con todos los documentos analizados, y donde podemos encontrar espacios de pertenencia, como lo es el caso de la relación entre ideología y universidad o de causalidad como lo es el de ideología y el conocimiento y por supuesto las asociaciones ya vistas entre poder, conocimiento y universidad. ¿Qué 
significan estos niveles de asociación? Cualitativamente nos indican que el conocimiento es una causa de la ideología lo que podría contrastarse con la asociación que se hace con el poder, es decir, existen lógicas de poder que regulan el conocimiento y que este, a su vez, genera lo elementos prácticos y teóricos de una ideología, sin olvidar que esta última es un sistema de creencias. También nos indica, gracias al código de universidad que en este caso fue utilizado para visibilizar la identidad con la que las universidades se presentan a la comunidad, que la institución universitaria se instala en un campo definido por las lógicas de poder y las lógicas de ideología, cuya representación se ha observado atentamente en los anteriores análisis (ver figura 4).

Figura 4. Cartografía de densidad asociativa general mediante análisis de unidades hermenéuticas

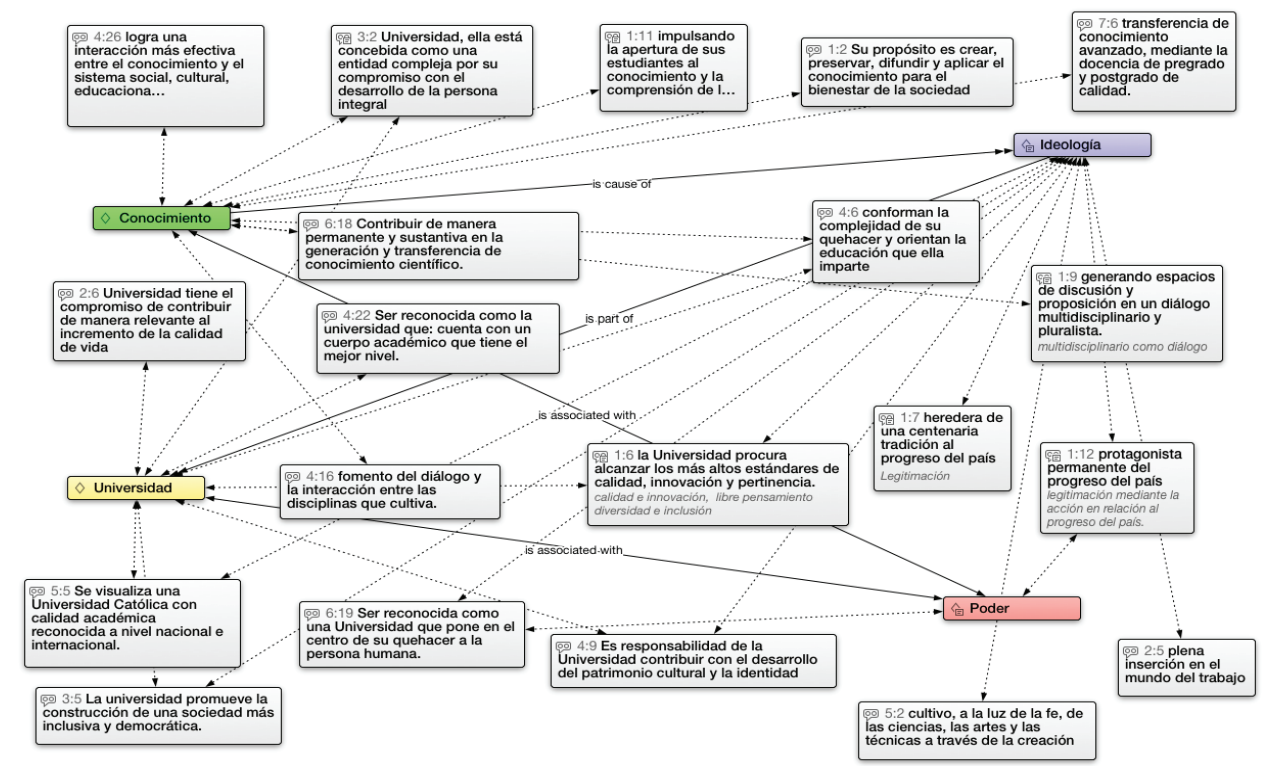

Fuente: elaboración propia

\section{CONCLUSIONES GENERALES Y LIMITACIONES/PROYECCIONES DE LA INVESTIGACIÓN}

\subsection{CONCLUSIONES GENERALES}

\subsubsection{La Educación Superior se rige bajo parámetros económicos y, por tanto, la pertinen-} cia del conocimiento como concepto debe colocarse en cuestión con mayor profundidad

Es pertinente y necesario continuar con indagaciones sobre cómo afecta el sistema económico a la concepción de conocimiento en las instituciones de hoy. Nos arriesgamos a concebir un conocimiento vacío basado en nociones de calidad, desarrollo e innovación 
confusas y orientadas solo a la productividad y la formación de cuerpos dóciles obedientes a la maquinaria social. Esta investigación contribuye a una discusión sobre los fundamentos epistemológicos de la universidad hoy en día y busca ampliar la noción de conocimiento no solo desde el pragmatismo que significa la aplicación de un saber, sino de su expansión, de su crecimiento y sinergia en espacios no formales, con el propósito de garantizar un acceso al conocimiento no solo en la universidad, sino también desde sus actores y formados de la institución.

\subsubsection{La construcción y generación de conocimiento exigen mayores parámetros de trans- disciplinariedad y complejidad}

El estudio y conclusiones del Informe de la OECD han señalado que la pedagogía e investigación de la educación terciaria debe fomentar la relación de áreas diversas del conocimiento, en base a programas multi y transdisciplinarios más extensos y formas más complejas de crear conocimiento. Dichos desafíos involucran una redefinición del rol de las universidades y la educación terciaria en general como, asimismo, esquemas de identidad disciplinar y las dinámicas relacionales de sus participantes (OECD 2009). Esta investigación avala lo propuesto por la OECD sumando la necesidad de un conocimiento complejo que contribuya a establecer un concepto del conocimiento relacionado a lo social, integrado a la trama de la realidad y que estimule la proyección de los estudiantes hacia áreas de creatividad y generación de conocimiento en términos de apertura y no clausura disciplinar.

\subsubsection{La Formación de profesionales se orienta a la tecnocratización del conocimiento}

La aparición de un nuevo modelo económico y una mayor demanda de conocimiento manifestada por la población, genera la inclusión del saber a estructuras de producción económicas, donde la educación se reacomoda en la matriz social como un bien intercambiable por dinero, accesible en la medida que se posean los recursos, pero ya no limitándose (teóricamente) a las elites intelectuales, si no abriéndose en un rango más amplio a diferencia de los años anteriores donde se ofrecía como un privilegio más que un servicio; atendía a una exclusiva minoría, la de los herederos. De la misma manera, el conocimiento entra en un mercado de producción válido en la medida que obedezca a prioridades sociales, generando la disyuntiva de las universidades de incorporarse a estas nuevas redes de saber o aislarse junto a los paradigmas tradicionales universitarios.

\subsubsection{Espacio social como micropolítica}

En este estudio se plantea permanentemente que el espacio de lucha debe darse bajo términos micropolíticos. La individualidad del ser humano como elemento complejo, exige que genere espacios de resistencia a los elementos ideológicos que permean la sociedad y la universidad. Este espacio debe constituirse bajo la posibilidad de difuminar el ejercicio del poder desde la ideología en cualquier contexto. Por lo tanto, se establece que el nivel superior de educación se constituye como un espacio complejo y multidimensional permeado por la ideología, pero, a la vez, con la posibilidad de generar espacios de microresistencia ideológica. 


\section{REFERENCIAS BIBLIOGRÁFICAS}

Ávila-Fuenmayor, F. (2006). El concepto de poder en Michel Foucault. Telos, 8(2), 215-234.

Brunner, J. (2000). Globalización y el futuro de la educación: tendencias, desafío, estrategias. Seminario sobre Prospectiva de la Educación en la Región de América Latina y el Caribe UNESCO, Santiago de Chile.

Del Valle, N. (2012). Entre poder y resistencia. Tras los rastros de la política en Foucault. Revista Enfoques, X(17), 147-168.

Espina, M. (2007) Complejidad, transdisciplina y metodología de la investigación social. Utopía y Praxis latinoamericana, 12(38), 29-43.

Fairclough, N. (1995). Critical discourse analysis: The critical study of language. London, New York: Longman.

Flick, U. (2004). Introducción a la Investigación Cualitativa. Madrid: Editorial Morata.

Fonti, D. (2014). Críticos, orgánicos, corporativos. Reflexiones sobre las responsabilidades y funciones sociales del trabajo intelectual universitario. Psicoperspectivas, 13(1), 6-14.

Foucault, M. (1966). Las palabras y las cosas. Buenos Aires: Siglo XXI Editores. . (1998). Historia de la Sexualidad: la voluntad de saber. Madrid: Siglo XXI Editores. . (2001). Defender la sociedad: Curso en el Collège de France: (1975-1976). Buenos Aires: Fondo de Cultura Económica.

Gandarilla, J. (2010). La universidad entrando al siglo XXI por el laberinto de la complejidad. Revista Perfiles educativos. XXXII(127), 130-143.

Giraldo, G. (2005). Teoría de la complejidad y premisas de legitimidad en las políticas de educación superior. Revista Cinta de Moebio, 22, 46-72.

Jessop, B. (2008). El futuro del Estado capitalista. Madrid: Los libros de la Catarata.

Lemaitre, M. (2005). La calidad colonizada: universidad y globalización. Revista de la Educación Superior, XXXIV(1), 123-134.

Márquez (2007). La problematización del poder en Michel Foucault. Revista Círculo de Humanidades de la Universidad Autónoma Latinoamericana, 28, 100-115.

Miles, M., \& Huberman, A.M. (1994). Data management and analysis methods. In N.K. Denzin \& Y.S. Lincoln (Eds.), Handbook of qualitative research (pp. 429-444). London: Sage Publication.

Morín, E. (2001). Introducción al pensamiento complejo. Barcelona: Gedisa. . (2006a). El método. La naturaleza de la naturaleza. Madrid: Cátedra. . (2006b). El método. El conocimiento del conocimiento. Madrid: Cátedra.

OECD (2009) Revisión de Políticas Nacionales de Educación. La Educación Superior en Chile. Santiago de Chile. Banco Mundial OECD Publishing.

Ricoeur, P. (1994). Ideología y utopía. Barcelona: Gedisa Editorial.

Toledo, U. (2007). Realidades Múltiples y Mundos Sociales. Revista Cinta Moebio, 30, 211-244.

Unesco. (2011). Recorridos nacionales: rumbo a la Educación para el Desarrollo Sostenible. París:

Organización de las Naciones Unidas para la Educación, la Ciencia y la Cultura.

van Dijk, T. (1999). Ideología: una aproximación multidisciplinaria. Barcelona: Editorial Gedisa. . (2008). Discurso y Poder. Barcelona: Editorial Gedisa. . (2012). Discurso y Contexto. Una aproximación cognitiva. Barcelona: Editorial Gedisa.

Vasilachis, I. (2006). Estrategias de Investigación Cualitativa. Barcelona: Editorial Gedisa. 
